\title{
Theory for atomic diffusion on fixed and deformable crystal lattices
}

\author{
Eliot Fried \\ Department of Theoretical and Applied Mechanics \\ University of Illinois at Urbana-Champaign \\ Urbana, IL 61801-2935, USA \\ Shaun Sellers \\ School of Mathematics \\ University of East Anglia \\ Norwich NR4 7TJ, England
}

\begin{abstract}
We develop a theoretical framework for the diffusion of a single unconstrained species of atoms on a crystal lattice that provides a generalization of the classical theories of atomic diffusion and diffusion-induced phase separation to account for constitutive nonlinearities, external forces, and the deformation of the lattice. In this framework, we regard atomic diffusion as a microscopic process described by two independent kinematic variables: $(i)$ the atomic flux, which reckons the local motion of atoms relative to the motion of the underlying lattice, and ( $i i)$ the time-rate of the atomic density, which encompasses nonlocal interactions between migrating atoms and characterizes the kinematics of phase separation. We introduce generalized forces power-conjugate to each of these rates and require that these forces satisfy ancillary microbalances distinct from the conventional balance involving the forces that expend power over the rate at which the lattice deforms. A mechanical version of the second law, which takes the form of an energy imbalance accounting for all power expenditures (including those due to the atomic diffusion and phase separation), is used to derive restrictions on the constitutive equations. With these restrictions, the microbalance involving the forces conjugate to the atomic flux provides a generalization of the usual constitutive relation between the atomic flux and the gradient of the diffusion potential, a relation that in conjunction with the atomic balance yields a generalized Cahn-Hilliard equation.
\end{abstract}

Dedicated to Roger Fosdick, whose fundamental and broad-reaching work in continuum thermomechanics has provided us with abundant inspiration.

\section{Introduction}

The diffusion of a single unconstrained atomic species on a fixed crystal lattice is commonly modeled by the Cahn-Hilliard equation (see, for 
example, Cahn [1, 2, 3] and Cahn \& Hilliard [4, 5, 6])

$$
\dot{\nu}=\operatorname{Div}\left(D_{1}(\nu) \operatorname{Grad} \nu-D_{2}(\nu) \operatorname{Grad}(\operatorname{Div} \operatorname{Grad} \nu)\right),
$$

with $\nu$ the atomic density, $D_{1}$ the nonnegative density-dependent diffusivity, and $D_{2}$ a small, nonnegative density-dependent coefficient. The first term on the right side is due to simple diffusion, whereas the second one is a higher order term that takes into account spatial inhomogeneities on a fine scale, such as those that may arise during processes involving phase separation. This equation can be derived by adjoining to the atomic balance

$$
\dot{\nu}=-\operatorname{Div} \boldsymbol{\jmath}
$$

the constitutive relation

$$
\boldsymbol{\jmath}=-\kappa(\nu) \operatorname{Grad} \mu,
$$

determining the atomic flux as proportional to the gradient Grad $\mu$ of the diffusion potential $\mu$ through a nonnegative scalar mobility $\kappa$ that, in general, depends on the atomic density. In simple atomic diffusion, the diffusion potential is given by the derivative of a constitutive response function $\hat{\psi}$ that determines the energy density $\psi$ in terms of the atomic density, viz.,

$$
\mu=\hat{\psi}^{\prime}(\nu)
$$

in which case the diffusive atomic flux takes the form

$$
\boldsymbol{\jmath}=-D(\nu) \operatorname{Grad} \nu
$$

with diffusivity $D$ determined by

$$
D(\nu)=\kappa(\nu) \hat{\psi}^{\prime \prime}(\nu) .
$$

However, when strong spatial inhomogeneities in the density may be present, the response function determining the energy density is taken to depend on both the density $\nu$ and its gradient $\operatorname{Grad} \nu$, so that the diffusion potential is given by the variational derivative

$$
\mu=\frac{\partial \hat{\psi}}{\partial \nu}(\nu, \operatorname{Grad} \nu)-\operatorname{Div}\left(\frac{\partial \hat{\psi}}{\partial(\operatorname{Grad} \nu)}(\nu, \operatorname{Grad} \nu)\right) .
$$

Often, in this context, it is presumed that $\hat{\psi}$ has the particular form

$$
\hat{\psi}(\nu, \operatorname{Grad} \nu)=f(\nu)+\frac{1}{2} \alpha|\operatorname{Grad} \nu|^{2},
$$


with $\alpha$ a positive constant that is usually interpreted as proportional to a squared characteristic length of interaction and, hence, taken to be small, so that the atomic flux becomes

$$
\boldsymbol{\jmath}=-D_{1}(\nu) \operatorname{Grad} \nu+D_{2}(\nu) \operatorname{Grad}(\operatorname{Div} \operatorname{Grad} \nu),
$$

with coefficients

$$
D_{1}(\nu)=\kappa(\nu) f^{\prime \prime}(\nu) \quad \text { and } \quad D_{2}(\nu)=\alpha \kappa(\nu) .
$$

Recently, Gurtin [7] presented a thermodynamically consistent framework that provides a broad generalization of (1.1) that accounts for constitutive nonlinearities, kinetics, and deformation of the underlying lattice. This framework is based on the belief that to each independent kinematic process there should correspond a system of power-conjugate forces subject to a generalized force balance.] Specifically, Gurtin [7] identifies atomic diffusion and lattice deformation as distinct kinematical processes, with the density-rate $\dot{\nu}$ being a generalized velocity associated with atomic diffusion and the deformation-rate $\dot{\boldsymbol{y}}$ being the velocity of the underlying lattice. Associated with $\dot{\nu}$ is a system of generalized forces that satisfy a scalar microforce balance. This balance is a postulate distinct from and ancillary to the conventional force balance involving forces that act power conjugate to the velocity of the underlying lattice. With appropriate thermodynamically consistent constitutive equations, the microforce balance yields an expression for the diffusion potential that generalizes (1.7). Finally, the generalized Cahn-Hilliard equation arises on combining with the atomic balance the thermodynamically derived expression determining the atomic flux as proportional to the gradient of the diffusion potential.

Although this approach provides a powerful and appealing means of deriving (1.1) and various generalizations thereof, we believe that it is lacking in the sense that it accounts only for one aspect of the kinematics associated with atomic diffusion. In particular, we take the view that the diffusive flux of atoms, as described by $\boldsymbol{\jmath}$, should be considered as a kinematic process distinct from that described by the density-rate $\dot{\nu}$. Specifically, we conceive of atomic diffusion as a microscopic process described by

(i) the atomic flux $\boldsymbol{J}$, which measures the local motion of atoms relative to macroscopic motion of the lattice as characterized by the velocity $\dot{\boldsymbol{y}}$, and by

\footnotetext{
1 A foundation for this viewpoint can be devised using the principle of virtual power, see, for example, Germain [8], Antman \& Osborn [9], Maugin [10], and Frémond 11]. We follow here an alternative, but essentially equivalent, approach taken by Ericksen 12], Goodman \& Cowin 13], Capriz \& Podio-Guidugli 14], Capriz 15], Fried \& Gurtin 16, 17], Fried [18], and Gurtin [7].
} 
(ii) the rate $\dot{\nu}$ of the atomic density, which encompasses nonlocal interactions between diffusing atoms and characterizes the kinematics of phase separation.

Adopting the view promoted by Fried \& Gurtin [16, 17], Fried [18], and Gurtin [7], we arrive at an alternative derivation of the Cahn-Hilliard equation (1.1), where, to account for power expenditures associated with the microscopic motion of atoms, we introduce two separate systems of forces - one being power-conjugate to the atomic flux $\boldsymbol{J}$ and the other being power-conjugate to the rate $\dot{\nu}$ of the atomic density. Further, we require that these forces be consistent with separate microforce balances, one vectorial and involving the forces power-conjugate to $\boldsymbol{J}$ and the other scalar and involving the forces power-conjugate to $\dot{\nu}$.

We first focus on the development of a simple theory, where, to highlight the roles of the vectorial and scalar microforces, the lattice is fixed. Next, we extend the theory to include deformation of the lattice. For simplicity, we restrict our attention to processes involving only atomic diffusion and lattice deformation, ignoring thermal and other effects, so that the first and second laws of thermodynamics are replaced by an energy imbalance that accounts for all relevant power expenditures. As a further simplification, we ignore all forms of inertia. We employ a purely referential description and consistently use direct notation (see, for example, Gurtin [20]).

\section{Simple theory for atomic diffusion on fixed lattice}

\subsection{BALANCE AND IMBALANCE LAWS}

The theory is based upon the following laws:

- atomic balance;

- flux-conjugate microforce balance;

- density-rate-conjugate microforce balance;

- energy imbalance.

In contrast to standard formulations of diffusion in rigid bodies, we include in this list of balances two microforce balances, which will be

\footnotetext{
${ }^{2}$ Previously, Fried \& Sellers 19] developed a theory for solute transport in which the diffusive mass flux is interpreted as a generalized velocity and corresponding generalized forces subject to an ancillary force balance are introduced.
} 
seen to provide appropriate generalizations of commonly assumed constitutive relations. In particular, the flux-conjugate microforce balance provides the generalization of (1.3), whereas the density-rate-conjugate microforce balance provides the generalization of (1.7).

We articulate our basic laws of balance and imbalance in global form over a generic subregion $\mathcal{P}$ of the region $\mathcal{B}$ occupied by the body in a fixed reference configuration. The outward unit-normal field to $\partial \mathcal{P}$, directed outward from $\mathcal{P}$, is designated by $\boldsymbol{n}$.

\subsubsection{Atomic balance}

We introduce the fields

$$
\begin{array}{ll}
\nu & \text { atomic density, } \\
\boldsymbol{J} & \text { atomic flux, } \\
m & \text { external atomic supply, }
\end{array}
$$

in which case the integrals

$$
\int_{\mathcal{P}} \nu d v, \quad \int_{\partial \mathcal{P}} \boldsymbol{J} \cdot \boldsymbol{n} d a, \quad \text { and } \quad \int_{\mathcal{P}} m d v
$$

represent, respectively, the number of atoms in $\mathcal{P}$, the number of atoms, per unit time, added to $\mathcal{P}$ by diffusion across $\partial \mathcal{P}$, and the number of atoms added, per unit time, to $\mathcal{P}$ by agencies external to $\mathcal{P}$.

Atomic balance is the postulate that, for each region $\mathcal{P}$ and each instant, the rate at which the number of atoms within $\mathcal{P}$ changes with respect to time be equal to the rate at which atoms are added to $\mathcal{P}$ by the diffusion across $\partial \mathcal{P}$ and by external supplies to $\mathcal{P}$ :

$$
\overline{\int_{\mathcal{P}} \nu d v}=-\int_{\partial \mathcal{P}} \boldsymbol{J} \cdot \boldsymbol{n} d a+\int_{\mathcal{P}} m d v .
$$

Using the transport and divergence theorems, we obtain the equivalent local field equation

$$
\dot{\nu}=-\operatorname{Div} \boldsymbol{\jmath}+m
$$

enforcing atomic balance.

\footnotetext{
${ }^{3}$ The supply $m$ should not be confused with a reaction term. Rather, we view $m$ as an external supply that accounts for the insertion of material by an external observer. Its role is analogous to that of the external heat supply that is routinely included in modern continuum-thermodynamical statements of energy balance (see, for example, Truesdell [21]). By introducing the external mass supply $m$, we follow Gurtin [7].
} 
2.1.2. Flux-conjugate microforce balance

Associated with the evolution of the atomic flux $\boldsymbol{J}$, we introduce a system of power-conjugate microforces, consisting of

$$
\begin{array}{ll}
\boldsymbol{\Sigma} & \text { flux-conjugate microstress, } \\
\boldsymbol{h} & \text { flux-conjugate internal body microforce, } \\
\boldsymbol{\ell} & \text { flux-conjugate external body microforce, }
\end{array}
$$

in which case the integrals

$$
\int_{\mathcal{P}} \boldsymbol{\Sigma} \boldsymbol{n} d \mathrm{a}, \quad \int_{\mathcal{P}} \boldsymbol{h} d v, \quad \text { and } \quad \int_{\mathcal{P}} \ell d v
$$

represent the flux-conjugate microforces exerted on the region $\mathcal{P}$ by the flux-conjugate microtraction distributed over $\partial \mathcal{P}$, by agencies within $\mathcal{P}$, and by flux-conjugate agencies external to $\mathcal{P}$, respectively.

Flux-conjugate microforce balance is the postulate that, for each region $\mathcal{P}$ and each instant, the resultant, taking both internal and external sources into account, of the flux-conjugate microforces acting on $\mathcal{P}$ vanishes:

$$
\int_{\partial \mathcal{P}} \boldsymbol{\Sigma} \boldsymbol{n} d a+\int_{\mathcal{P}}(\boldsymbol{h}+\boldsymbol{\ell}) d v=\mathbf{0} .
$$

Using the divergence theorem, we arrive at the equivalent local field equation

$$
\operatorname{Div} \boldsymbol{\Sigma}+\boldsymbol{h}+\boldsymbol{\ell}=\mathbf{0}
$$

enforcing the flux-conjugate microforce balance.

\subsubsection{Density-rate-conjugate microforce balance}

Associated with the evolution of the atomic density $\nu$, we introduce a system of power-conjugate microforces, consisting of

$$
\begin{aligned}
& \boldsymbol{\xi} \text { density-rate-conjugate microstress vector, } \\
& \pi \text { density-rate-conjugate internal body microforce, } \\
& \gamma \text { density-rate-conjugate external body microforce, }
\end{aligned}
$$

in which case the integrals

$$
\int_{\mathcal{P}} \boldsymbol{\xi} \cdot \boldsymbol{n} d a, \quad \int_{\mathcal{P}} \pi d v, \quad \text { and } \quad \int_{\mathcal{P}} \gamma d v
$$


represent the density-rate-conjugate microforces exerted on the region $\mathcal{P}$ by the density-rate-conjugate microtraction distributed over $\partial \mathcal{P}$, by agencies within $\mathcal{P}$, and by density-rate-conjugate agencies external to $\mathcal{P}$, respectively.

Density-rate-conjugate microforce balance is the postulate that, for each region $\mathcal{P}$ and each instant, the resultant, taking both internal and external sources into account, of the density-rate-conjugate microforces acting on $\mathcal{P}$ vanishes:

$$
\int_{\partial \mathcal{P}} \boldsymbol{\xi} \cdot \boldsymbol{n} d a+\int_{\mathcal{P}}(\pi+\gamma) d v=0
$$

Using the divergence theorem, we arrive at the equivalent local field equation

$$
\operatorname{Div} \boldsymbol{\xi}+\pi+\gamma=0
$$

enforcing the density-rate-conjugate microforce balance.

\subsubsection{Energy imbalance}

To formulate this imbalance, we introduce fields

$$
\begin{aligned}
& \psi \quad \text { free energy density, } \\
& \mu \quad \text { diffusion potential, }
\end{aligned}
$$

in which case the integrals

$$
\int_{\mathcal{P}} \psi d v \quad \text { and } \quad \int_{\mathcal{P}} \mu m d v
$$

represent the free energy in $\mathcal{P}$ and rate at which energy is added to $\mathcal{P}$ through the external supply of atoms to $\mathcal{P}$.

Further, the integrals

$$
\int_{\partial \mathcal{P}}\left(\boldsymbol{\Sigma}^{\top} \boldsymbol{\jmath}+\boldsymbol{\xi} \dot{\nu}\right) \cdot \boldsymbol{n} d a \quad \text { and } \quad \int_{\mathcal{P}}(\boldsymbol{\ell} \cdot \boldsymbol{\jmath}+\gamma \dot{\nu}) d v
$$

provide an accounting of the power expended on $\mathcal{P}$ by the tractions acting on $\partial \mathcal{P}$ and by the agencies acting external to that region. 
Energy imbalance is the postulate that, for each region $\mathcal{P}$ and each instant:用

$$
\overline{\int_{\mathcal{P}} \psi d v} \leq \int_{\partial \mathcal{P}}\left(\boldsymbol{\Sigma}^{\top} \boldsymbol{J}+\boldsymbol{\xi} \dot{\nu}\right) \cdot \boldsymbol{n} d a+\int_{\mathcal{P}}(\boldsymbol{\ell} \cdot \boldsymbol{J}+\gamma \dot{\nu}+\mu m) d v .
$$

Using the transport and divergence theorem as before, we arrive at the local inequality

$$
-\dot{\psi}+(\mu-\pi) \dot{\nu}+\boldsymbol{\xi} \cdot \operatorname{Grad} \dot{\nu}-\boldsymbol{h} \cdot \boldsymbol{\jmath}+(\boldsymbol{\Sigma}+\mu \boldsymbol{1}) \cdot \operatorname{Grad} \boldsymbol{\jmath} \geq 0
$$

enforcing energy imbalance.

\subsection{Constitutive equations}

We assume that the free energy density $\psi$, the flux-congugate microstress $\boldsymbol{\Sigma}$, the flux-congugate internal body microforce $\boldsymbol{h}$, the densityrate-conjugate microstress $\boldsymbol{\xi}$, and the density-rate-conjugate internal scalar body microforce $\pi$ are determined constitutively in terms of the atomic density $\nu$, the gradient Grad $\nu$ of the atomic density, the diffusion potential $\mu$, the gradient $\operatorname{Grad} \mu$ of the diffusion potential, and the atomic flux $\boldsymbol{g}$ :

$$
\left.\begin{array}{rl}
\psi & =\hat{\psi}(\nu, \operatorname{Grad} \nu, \mu, \operatorname{Grad} \mu, \boldsymbol{\jmath}), \\
\boldsymbol{\Sigma} & =\hat{\boldsymbol{\Sigma}}(\nu, \operatorname{Grad} \nu, \mu, \operatorname{Grad} \mu, \boldsymbol{\jmath}), \\
\boldsymbol{h} & =\hat{\boldsymbol{h}}(\nu, \operatorname{Grad} \nu, \mu, \operatorname{Grad} \mu, \boldsymbol{\jmath}), \\
\boldsymbol{\xi} & =\hat{\boldsymbol{\xi}}(\nu, \operatorname{Grad} \nu, \mu, \operatorname{Grad} \mu, \boldsymbol{\jmath}), \\
\pi & =\hat{\pi}(\nu, \operatorname{Grad} \nu, \mu, \operatorname{Grad} \mu, \boldsymbol{\jmath}) .
\end{array}\right\}
$$

We emphasize that, in contrast to the standard approach to the theory of atomic diffusion, neither the diffusion potential $\mu$ nor the atomic flux $\boldsymbol{\jmath}$ is given by a constitutive relation.

Inserting the constitutive relations (2.14) into the local energy imbalance (2.13) and writing, for brevity,

$$
z=(\nu, \operatorname{Grad} \nu, \mu, \operatorname{Grad} \mu, \boldsymbol{\jmath}),
$$

\footnotetext{
${ }^{4}$ To account for the diffusive flux of energy, Gurtin [f] included in his statement of energy imbalance the term$$
-\int_{\partial \mathcal{P}} \mu \boldsymbol{J} \cdot \boldsymbol{n} d a .
$$

Since, in our context, the diffusive flux of energy can be interpreted as the surface power of the atomic diffusion, our theory accounts for this effect through the term with integrand $\boldsymbol{\Sigma}^{\top} \boldsymbol{J} \cdot \boldsymbol{n}$.
} 
we arrive at the functional inequality

$$
\begin{aligned}
& \left(\frac{\partial \hat{\psi}}{\partial \nu}(z)-\mu+\hat{\pi}(z)\right) \dot{\nu}+\left(\frac{\partial \hat{\psi}}{\partial(\operatorname{Grad} \nu)}(z)-\hat{\boldsymbol{\xi}}(z)\right) \cdot \operatorname{Grad} \dot{\nu}+\frac{\partial \hat{\psi}}{\partial \mu}(z) \dot{\mu} \\
+ & \frac{\partial \hat{\psi}}{\partial(\operatorname{Grad} \mu)}(z) \cdot \operatorname{Grad} \dot{\mu}+\frac{\partial \hat{\psi}}{\partial \boldsymbol{\jmath}}(z) \cdot \boldsymbol{j}+\hat{\boldsymbol{h}}(z) \cdot \boldsymbol{\jmath}-(\hat{\boldsymbol{\Sigma}}(z)+\mu \boldsymbol{1}) \cdot \operatorname{Grad} \boldsymbol{\jmath} \leq 0 .
\end{aligned}
$$

Hence, following the procedure founded by Coleman \& Noll [22] in their incorporation of the second law into continuum thermomechanics, we find that:

(i) the constitutive response functions $\hat{\psi}$ and $\hat{\boldsymbol{\xi}}$ delivering the free energy density $\psi$ and the density-rate-conjugate microstress $\boldsymbol{\xi}$ must be independent of the diffusion potential $\mu$, the gradient Grad $\mu$ of the diffusion potential, and the atomic flux $\boldsymbol{\jmath}$, and obey

$$
\hat{\boldsymbol{\xi}}(\nu, \operatorname{Grad} \nu)=\frac{\partial \hat{\psi}}{\partial(\operatorname{Grad} \nu)}(\nu, \operatorname{Grad} \nu)
$$

(ii) the constitutive response function $\hat{\boldsymbol{\Sigma}}$ delivering the flux-conjugate microstress $\Sigma$ must be independent of the atomic density $\nu$, the gradient Grad $\nu$ of the atomic density, the gradient Grad $\mu$ of the diffusion potential, and the atomic flux $\boldsymbol{J}$, and obey

$$
\hat{\Sigma}=-\mu 1
$$

(iii) the constitutive response function $\hat{\pi}$ delivering the density-rateconjugate internal body microforce $\pi$ must be independent of the gradient Grad $\mu$ of the diffusion potential and the atomic flux $\boldsymbol{\jmath}$, and obey

$$
\hat{\pi}(\nu, \operatorname{Grad} \nu, \mu)=\mu-\frac{\partial \hat{\psi}}{\partial \nu}(\nu, \operatorname{Grad} \nu)
$$

(iv) the constitutive response function $\hat{\boldsymbol{h}}$ for the flux-conjugate internal body microforce $\boldsymbol{h}$ must be consistent with the residual inequality

$$
\hat{\boldsymbol{h}}(\nu, \operatorname{Grad} \nu, \mu, \operatorname{Grad} \mu, \boldsymbol{\jmath}) \cdot \boldsymbol{\jmath} \leq 0 .
$$

Further, granted smoothness of the response function $\hat{\boldsymbol{h}}$, a result due to Gurtin \& Voorhees [23] yields a general solution

$$
\boldsymbol{h}=-\boldsymbol{Z}(\nu, \operatorname{Grad} \nu, \mu, \operatorname{Grad} \mu, \boldsymbol{\jmath}) \boldsymbol{\jmath}
$$


of $(2.20)$, where the reciprocal mobility tensor $\boldsymbol{Z}$ must obey

$$
\boldsymbol{\jmath} \cdot \boldsymbol{Z}(\nu, \operatorname{Grad} \nu, \mu, \operatorname{Grad} \mu, \boldsymbol{\jmath}) \boldsymbol{\jmath} \geq 0 .
$$

The governing equations that arise on substituting the foregoing thermodynamically consistent constitutive equations in the local field equations (2.9) and (2.6) expressing flux-conjugate microforce balance and density-rate-conjugate microforce balance read

$$
\left.\begin{array}{r}
\boldsymbol{Z}(\nu, \operatorname{Grad} \nu, \mu, \operatorname{Grad} \mu, \boldsymbol{\jmath}) \boldsymbol{\jmath}+\operatorname{Grad} \mu=\boldsymbol{\ell}, \\
\frac{\partial \hat{\psi}}{\partial \nu}(\nu, \operatorname{Grad} \nu)-\operatorname{Div}\left(\frac{\partial \hat{\psi}}{\partial(\operatorname{Grad} \nu)}(\nu, \operatorname{Grad} \nu)\right)-\mu=\gamma .
\end{array}\right\}
$$

Together with the atomic balance (2.3), (2.23) form the final governing equations of our theory for the diffusion of a single unconstrained atomic species on a fixed lattice.

The foregoing results show that the behavior of a medium of the sort considered here is completely determined by the provision of two constitutive response functions:

- $\hat{\psi}$ determining the free energy density as a function of the density $\nu$ and its gradient $G r a d \nu$; and

- the reciprocal mobility tensor $\boldsymbol{Z}$, which, in general, may depend on $\nu, \operatorname{Grad} \nu, \mu, \operatorname{Grad} \mu$, and $\boldsymbol{\jmath}$.

Provided that the external supplies $m, \boldsymbol{\ell}$, and $\gamma$ all vanish, that the constitutive function $\hat{\psi}$ is quadratic in the gradient of the atomic density and twice differentiable with respect to the atomic density, and that $\boldsymbol{Z}=\zeta \boldsymbol{1}$ with $\zeta$ depending at most on the atomic density, the system formed by combining (2.23) with the atomic balance (2.3) yields precisely the Cahn-Hilliard equation (1.1). If, furthermore, $\hat{\psi}$ is independent of the gradient of the atomic density, we obtain

$$
\boldsymbol{\jmath}=-\frac{\hat{\psi}^{\prime \prime}(\nu)}{\zeta(\nu)} \operatorname{Grad} \nu,
$$

for the atomic flux $\boldsymbol{\jmath}$ in terms of the density $\nu$ and its gradient Grad $\nu$. Comparing (2.24) with the conventional relation (1.5), we obtain an expression

$$
D(\nu)=\frac{\hat{\psi}^{\prime \prime}(\nu)}{\zeta(\nu)}
$$

for the diffusivity. Hence, within our framework, a constitutive relation of the form (1.5) can, therefore, be interpreted as an expression of fluxconjugate microforce balance, granted that the flux-conjugate internal 
body microforce is isotropic and linear in the atomic flux and that the flux-conjugate external body microforce vanishes. If, moreover, we assume that $\hat{\psi}$ has the particular form

$$
\hat{\psi}(\nu)=k_{\mathrm{B}} \theta \nu \log \nu
$$

corresponding to the classical entropic contribution to the free energy of a dilute mixture, with $k_{\mathrm{B}}$ Boltzmann's constant and $\theta$ the absolute temperature, then (2.25) reduces to

$$
D(\nu)=\frac{k_{\mathrm{B}} \theta}{\zeta(\nu)}
$$

the standard relation between diffusivity and mobility (see, for example, Nernst [24] and Einstein [25]). More generally, granted that the reciprocal mobility tensor $\boldsymbol{Z}$ is invertible, our theory in conjunction with the assumption (2.26) yields the diffusivity as a tensor

$$
\boldsymbol{D}(\nu, \operatorname{Grad} \nu, \mu, \operatorname{Grad} \mu, \boldsymbol{\jmath})=k_{\mathrm{B}} \theta \boldsymbol{Z}^{-1}(\nu, \operatorname{Grad} \nu, \mu, \operatorname{Grad} \mu, \boldsymbol{\jmath}) .
$$

\section{Theory accounting for both atomic diffusion and lattice deformation}

\subsection{BALANCE AND IMBALANCE LAWS}

In addition to the mass balance (2.2), flux-conjugate microforce balance (2.5), and density-rate-conjugate microforce balance (2.8), the theory is based upon the following laws:

- lattice-velocity-conjugate force balance;

- lattice-velocity-conjugate torque balance;

- energy imbalance.

Again, the standard formulation of diffusion in a deformable body is supplemented with two microforce balances, which will provide appropriate generalizations of (1.3) and (1.7).

\subsubsection{Lattice-velocity-conjugate force balance and lattice-velocity-conjugate torque balance}

The motion of the lattice is described by a mapping $\boldsymbol{y}$ of points $\boldsymbol{x}$ in $\mathcal{B}$ and times $t$ into points $\boldsymbol{y}(\boldsymbol{x}, t)$ of space. We require that $\operatorname{Det}(\operatorname{Grad} \boldsymbol{y})>$ 0 . Associated with this motion, we introduce a system of power-conjugate forces consisting of 


$$
\begin{aligned}
& \boldsymbol{S} \text { lattice-velocity-conjugate (Piola) stress, } \\
& \boldsymbol{b} \text { lattice-velocity-conjugate body force, }
\end{aligned}
$$

in which case the integrals

$$
\int_{\mathcal{P}} \boldsymbol{S n} d a \quad \text { and } \quad \int_{\mathcal{P}} \boldsymbol{b} d v
$$

represent the lattice-velocity-conjugate forces exerted on the region $\mathcal{P}$ by the lattice-velocity-conjugate traction distributed over $\partial \mathcal{P}$ and by lattice-velocity-conjugate agencies external to $\mathcal{P}$, respectively. Further, the integrals

$$
\int_{\mathcal{P}} \boldsymbol{y} \times \boldsymbol{S n} d a \quad \text { and } \quad \int_{\mathcal{P}} \boldsymbol{y} \times \boldsymbol{b} d v
$$

represent the lattice-velocity-conjugate torques exerted on the region $\mathcal{P}$ by the lattice-velocity-conjugate traction distributed over $\partial \mathcal{P}$ and by lattice-velocity-conjugate agencies external to $\mathcal{P}$, respectively.

Lattice-velocity-conjugate force balance is the postulate that, for each region $\mathcal{P}$ and each instant, the resultant of the lattice-velocity-conjugate forces acting on $\mathcal{P}$ vanishes:

$$
\int_{\partial \mathcal{P}} \boldsymbol{S n} d a+\int_{\mathcal{P}} \boldsymbol{b} d v=\mathbf{0} .
$$

Further, lattice-velocity-conjugate torque balance is the postulate that, for each region $\mathcal{P}$ and each instant, the resultant of the torques associated with the lattice-velocity-conjugate forces acting on $\mathcal{P}$ vanishes:

$$
\int_{\partial \mathcal{P}} \boldsymbol{y} \times \boldsymbol{S n} d a+\int_{\mathcal{P}} \boldsymbol{y} \times \boldsymbol{b} d v=\mathbf{0} .
$$

In writing (3.4), we have assumed that the diffusing atoms do not induce torques, as would be expected at least for approximately spherical atoms. Using the divergence theorem, we arrive at the equivalent local field equations

$$
\operatorname{Div} \boldsymbol{S}+\boldsymbol{b}=\mathbf{0} \quad \text { and } \quad(\operatorname{Grad} \boldsymbol{y}) \boldsymbol{S}^{\top}=\boldsymbol{S}(\operatorname{Grad} \boldsymbol{y})^{\top} .
$$




\subsubsection{Energy imbalance}

To account for power expenditures associated with the motion of the lattice, we modify the energy imbalance (2.12) to read

$$
\overline{\int_{\mathcal{P}} \psi d v} \leq \int_{\partial \mathcal{P}}\left(\boldsymbol{\Sigma}^{\top} \boldsymbol{J}+\boldsymbol{\xi} \dot{\nu}+\boldsymbol{S}^{\top} \dot{\boldsymbol{y}}\right) \cdot \boldsymbol{n} d a+\int_{\mathcal{P}}(\boldsymbol{l} \cdot \boldsymbol{\jmath}+\pi \dot{\nu}+\mu m+\boldsymbol{b} \cdot \dot{\boldsymbol{y}}) d v
$$

Proceeding as before, we arrive at the local inequality

$$
\begin{aligned}
&-\dot{\psi}+(\mu-\pi) \dot{\nu}+\boldsymbol{\xi} \cdot \operatorname{Grad} \dot{\nu}-\boldsymbol{h} \cdot \boldsymbol{\jmath} \\
&+\boldsymbol{S} \cdot \operatorname{Grad} \dot{\boldsymbol{y}}+(\boldsymbol{\Sigma}+\mu \mathbf{1}) \cdot \operatorname{Grad} \boldsymbol{\jmath} \geq 0 .
\end{aligned}
$$

\subsection{Constitutive EQuations}

We add the lattice-velocity-conjugate stress and the lattice-motion gradient Grad $\boldsymbol{y}$ to the lists of dependent and independent constitutive variables, respectively, so that

$$
\left.\begin{array}{rl}
\psi & =\hat{\psi}(\nu, \operatorname{Grad} \nu, \mu, \operatorname{Grad} \mu, \boldsymbol{\jmath}, \operatorname{Grad} \boldsymbol{y}), \\
\boldsymbol{\Sigma} & =\hat{\boldsymbol{\Sigma}}(\nu, \operatorname{Grad} \nu, \mu, \operatorname{Grad} \mu, \boldsymbol{\jmath}, \operatorname{Grad} \boldsymbol{y}), \\
\boldsymbol{h} & =\hat{\boldsymbol{h}}(\nu, \operatorname{Grad} \nu, \mu, \operatorname{Grad} \mu, \boldsymbol{\jmath}, \operatorname{Grad} \boldsymbol{y}), \\
\boldsymbol{\xi} & =\hat{\boldsymbol{\xi}}(\nu, \operatorname{Grad} \nu, \mu, \operatorname{Grad} \mu, \boldsymbol{\jmath}, \operatorname{Grad} \boldsymbol{y}), \\
\pi & =\hat{\pi}(\nu, \operatorname{Grad} \nu, \mu, \operatorname{Grad} \mu, \boldsymbol{\jmath}, \operatorname{Grad} \boldsymbol{y}), \\
\boldsymbol{S} & =\hat{\boldsymbol{S}}(\nu, \operatorname{Grad} \nu, \mu, \operatorname{Grad} \mu, \boldsymbol{\jmath}, \operatorname{Grad} \boldsymbol{y}) .
\end{array}\right\}
$$

Inserting the constitutive relations (3.8) into the local energy imbalance (3.7) and writing

$$
z=(\nu, \operatorname{Grad} \nu, \mu, \operatorname{Grad} \mu, \boldsymbol{\jmath}, \operatorname{Grad} \boldsymbol{y}),
$$

we arrive at the functional inequality

$$
\begin{gathered}
\left(\frac{\partial}{\partial \nu} \hat{\psi}(z)-\mu+\hat{\pi}(z)\right) \dot{\nu}+\left(\frac{\partial \hat{\psi}}{\partial(\operatorname{Grad} \nu)}(z)-\hat{\boldsymbol{\xi}}(z)\right) \cdot \operatorname{Grad} \dot{\nu}+\frac{\partial \hat{\psi}}{\partial \mu}(z) \dot{\mu} \\
\left.+\frac{\partial \hat{\psi}}{\partial(\operatorname{Grad} \mu)}(z) \cdot \operatorname{Grad} \dot{\mu}+\left(\frac{\partial \hat{\psi}}{\partial(\operatorname{Grad} \boldsymbol{y})}\right)(z)-\hat{\boldsymbol{S}}(z)\right) \cdot \operatorname{Grad} \dot{\boldsymbol{y}} \\
\quad+\frac{\partial \hat{\psi}}{\partial \boldsymbol{\jmath}}(z) \cdot \boldsymbol{j}+\hat{\boldsymbol{h}}(z) \cdot \boldsymbol{\jmath}-(\hat{\boldsymbol{\Sigma}}(z)+\mu \mathbf{1}) \cdot \operatorname{Grad} \boldsymbol{\jmath} \leq 0 .
\end{gathered}
$$

Hence, proceeding as before, we obtain the results 
(i) the constitutive response functions $\hat{\psi}, \hat{\boldsymbol{S}}$, and $\hat{\boldsymbol{\xi}}$ delivering the energy density $\psi$, the lattice-velocity-conjugate stress $\boldsymbol{S}$, and the density-rate-conjugate microstress $\boldsymbol{\xi}$ must be independent of the diffusion potential $\mu$, the gradient Grad $\mu$ of the diffusion potential, and the atomic flux $\boldsymbol{\jmath}$, and obey

$$
\left.\begin{array}{rl}
\hat{\boldsymbol{S}}(\nu, \operatorname{Grad} \nu, \operatorname{Grad} \boldsymbol{y}) & =\frac{\partial \hat{\psi}}{\partial(\operatorname{Grad} \boldsymbol{y})}(\nu, \operatorname{Grad} \nu, \operatorname{Grad} \boldsymbol{y}), \\
\hat{\boldsymbol{\xi}}(\nu, \operatorname{Grad} \nu, \operatorname{Grad} \boldsymbol{y}) & =\frac{\partial \hat{\psi}}{\partial(\operatorname{Grad} \nu)}(\nu, \operatorname{Grad} \nu, \operatorname{Grad} \boldsymbol{y}) ;
\end{array}\right\}
$$

(ii) the constitutive response function $\hat{\boldsymbol{\Sigma}}$ delivering the flux-conjugate microstress $\Sigma$ must be independent of the atomic density $\nu$, the gradient Grad $\nu$ of the atomic density, the gradient Grad $\mu$ of the diffusion potential, and the atomic flux $\boldsymbol{\jmath}$, and obey

$$
\hat{\Sigma}=-\mu 1
$$

(iii) the constitutive response function $\hat{\pi}$ delivering the density-rateconjugate internal body microforce $\pi$ must be independent of the gradient Grad $\mu$ of the diffusion potential and the atomic flux $\boldsymbol{\jmath}$, and obey

$$
\hat{\pi}(\nu, \operatorname{Grad} \nu, \mu)=\mu-\frac{\partial \hat{\psi}}{\partial \nu}(\nu, \operatorname{Grad} \nu)
$$

(iv) the constitutive response function $\hat{\boldsymbol{h}}$ for the flux-conjugate internal body microforce $\boldsymbol{h}$ must be consistent with the residual inequality

$$
\hat{\boldsymbol{h}}(\nu, \operatorname{Grad} \nu, \mu, \operatorname{Grad} \mu, \boldsymbol{\jmath}, \operatorname{Grad} \boldsymbol{y}) \cdot \boldsymbol{\jmath} \leq 0 .
$$

Analogous to the result (2.21), we have the general solution

$$
\boldsymbol{h}=-\boldsymbol{Z}(\nu, \operatorname{Grad} \nu, \mu, \operatorname{Grad} \mu, \boldsymbol{\jmath}, \operatorname{Grad} \boldsymbol{y}) \boldsymbol{\jmath}
$$

of (3.14), where the reciprocal mobility tensor $\boldsymbol{Z}$ must obey

$$
\boldsymbol{\jmath} \cdot \boldsymbol{Z}(\nu, \operatorname{Grad} \nu, \mu, \operatorname{Grad} \mu, \boldsymbol{\jmath}) \boldsymbol{\jmath} \geq 0 .
$$

Further, the constitutive relations are restricted by objectivity; that is, under the observer transformation

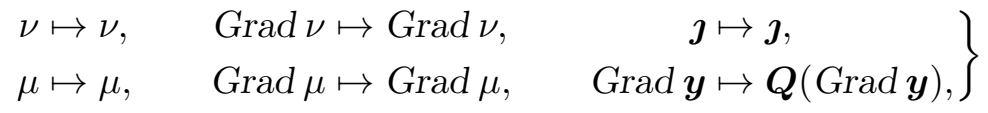


with $\boldsymbol{Q}$ orthogonal, we must have

$$
\left.\begin{array}{rlrlrl}
\boldsymbol{\Sigma} & \mapsto \boldsymbol{Q} \boldsymbol{\Sigma}, & & \boldsymbol{h} \mapsto \boldsymbol{h}, & & \boldsymbol{\ell} \mapsto \boldsymbol{\ell}, \\
\boldsymbol{\xi} & \mapsto \boldsymbol{\xi}, & & \pi \mapsto \pi, & & \gamma \mapsto \gamma, \\
\boldsymbol{S} & \mapsto \boldsymbol{Q} \boldsymbol{S}, & \boldsymbol{b} \mapsto \boldsymbol{b}, & & \psi \mapsto \psi .
\end{array}\right\}
$$

Granted the foregoing thermodynamic results, these transformations require, in particular, that

$$
\left.\begin{array}{rl}
\psi & =\tilde{\psi}(\nu, \operatorname{Grad} \nu, \boldsymbol{C}), \\
\boldsymbol{Z} & =\tilde{\boldsymbol{Z}}(\nu, \operatorname{Grad} \nu, \mu, \operatorname{Grad} \mu, \boldsymbol{\jmath}, \boldsymbol{C}), \\
\boldsymbol{S} & =(\operatorname{Grad} \boldsymbol{y}) \frac{\partial \tilde{\psi}}{\partial \boldsymbol{C}}(\nu, \operatorname{Grad} \nu, \boldsymbol{C}),
\end{array}\right\}
$$

with $\boldsymbol{C}=(\operatorname{Grad} \boldsymbol{y})^{\top} \operatorname{Grad} \boldsymbol{y}$ the right Cauchy-Green tensor.

The governing equations that arise on substituting the foregoing thermodynamically consistent constitutive equations in the local field equations $(3.5)_{1},(2.9)$ and $(2.6)$ expressing lattice-velocity-conjugate force balance, flux-conjugate microforce balance, and density-rate-conjugate microforce balance read

$$
\left.\begin{array}{r}
\operatorname{Div}\left((\operatorname{Grad} \boldsymbol{y}) \frac{\partial \tilde{\psi}}{\partial \boldsymbol{C}}(\nu, \operatorname{Grad} \nu, \boldsymbol{C})\right)+\boldsymbol{b}=\mathbf{0}, \\
\tilde{\boldsymbol{Z}}(\nu, \operatorname{Grad} \nu, \mu, \operatorname{Grad} \mu, \boldsymbol{\jmath}, \boldsymbol{C}) \boldsymbol{\jmath}+\operatorname{Grad} \mu=\boldsymbol{\ell}, \\
\frac{\partial \tilde{\psi}}{\partial \nu}(\nu, \operatorname{Grad} \nu, \boldsymbol{C})-\operatorname{Div}\left(\frac{\partial \tilde{\psi}}{\partial(\operatorname{Grad} \nu)}(\nu, \operatorname{Grad} \nu, \boldsymbol{C})\right)-\mu=\gamma .
\end{array}\right\}
$$

Together with the atomic balance (2.3), (3.20) form the final governing equations of our theory for the diffusion of a single unconstrained atomic species on a deformable lattice lattice.

We find that the behavior of a medium of the sort considered here is still completely determined by the provision of two constitutive response functions:

- $\tilde{\psi}$ determining the energy density as a function of the density $\nu$, the gradient Grad $\nu$ of the atomic density, and right Cauchy-Green tensor $\boldsymbol{C}$ associated with the lattice deformation; and

- the reciprocal mobility tensor $\tilde{\boldsymbol{Z}}$, which, in general, may depend on the atomic density $\nu$, the gradient Grad $\nu$ of the atomic density, the diffusion potential $\mu$, the gradient Grad $\mu$ of the diffusion potential, 
the atomic flux $\boldsymbol{J}$, and the right Cauchy-Green tensor $\boldsymbol{C}$ associated with the lattice deformation.

\section{Discussion}

Our theory is based upon introducing generalized velocities for diffusion and corresponding power-conjugate forces, which we call microforces, into an otherwise conventional continuum-mechanical description. In particular, we identify two distinct generalized velocities - a vectorvalued diffusive atomic flux that characterizes simple diffusion and a scalar-valued atomic density rate that characterizes phase separation. A systematic, thermodynamically consistent derivation generates a final system of governing equations - (2.3) and (2.23) for a fixed lattice, and (2.3) and (3.20) for a deformable lattice - that constitutes a generalization of common diffusion equations, a generalization that accounts for constitutive nonlinearities and external forces acting on the diffusing atoms.

We find that the constitutive response of the material is determined by the provision of two functions, one for the energy density and the other for the reciprocal mobility tensor. In particular, the Cahn-Hilliard equation (1.1) results on assuming that the energy response function is quadratic in Grad $\nu$ and that the reciprocal mobility tensor is proportional to the identity tensor by a scalar coefficient that is at most a function of $\nu$. Importantly, we arrive at our theory without introducing a constitutive relation such as (1.3) for the diffusive flux.

Additionally, our proposed format also leads to a generalization, (2.25), of the classical relation between diffusion coefficient and viscosity. We find that the classical form for the relation (2.27) holds when the free energy is given by the special constitutive relation (2.26).

A distinction between the final governing equations of our theory and those arising in more conventional derivations is that, in our approach, a vector-valued microforce balance provides a generalization between diffusive atomic flux and gradient of the diffusion potential.

\section{Acknowledgements}

This work was performed with financial support from the U. S. Department of Energy and the EPSRC (U.K.). 


\section{References}

1. J. W. Cahn, Free energy of a nonuniform system. II. Thermodynamic basis, Journal of Chemical Physics 30 (1959) 1121-1124.

2. J. W. Cahn, On spinodal decomposition, Acta Metallurgica 9 (1961) 795-801.

3. J. W. Cahn, On spinodal decomposition in cubic crystals, Acta Metallurgica 10 (1962) 179-183.

4. J. W. Cahn \& J. E. Hilliard, Free energy of a nonuniform system. I. Interfacial free energy, Journal of Chemical Physics 28 (1958) 258-267.

5. J. W. Cahn \& J. E. Hilliard, Free energy of a nonuniform system. III. Nucleation in a two-component incompressible fluid, Journal of Chemical Physics 31 (1959) 688-699.

6. J. W. Cahn \& J. E. Hilliard, Spinodal decomposition: a reprise, Acta Metallurgica 19 (1971) 151-161.

7. M. E. Gurtin, Generalized Ginzburg-Landau and Cahn-Hilliard equations based on a microforce balance, Physica D 92 (1996) 178-192.

8. P. Germain, Sur l'application de la méthode des puissances virtuelles en mécanique des milieux continus, Comptes Rendus de l'Académie des Sciences, série A 274 (1973) 1051-1055.

9. S. S. Antman \& J. E. Osborn, The principle of virtual work and integral laws of motion, Archive for Rational Mechanics and Analysis 69 (1979) 231-262.

10. G. A. Maugin, The principle of virtual power in continuum mechanicsapplication to coupled fields, Acta Mechanica 35 (1980) 1-70.

11. M. Frémond, Endommagement et principe des puissances virtuelles, Comptes Rendus de l'Académie des Sciences, série II 317 (1993) 857-863.

12. J. L. Ericksen, Conservation laws for liquid crystals, Transactions of the Society of Rheology 5 (1961) 23-34.

13. M. A. Goodman \& S. C. Cowin, A continuum theory for granular materials, Archive for Rational Mechanics and Analysis 44 (1972) 249-266.

14. G. Capriz \& P. Podio-Guidugli, Structured continua from a Lagrangian point of view, Annali di Matematica Pura ed Applicata 135 (1983) 1-25.

15. G. Capriz, Continua with Microstructure. New York: Springer-Verlag, 1989.

16. E. Fried \& M.E. Gurtin, Continuum theory of thermally induced phase transitions based on an order parameter, Physica D 68 (1993) 326-343.

17. E. Fried \& M. E. Gurtin, Dynamic solid-solid transitions with phase characterized by an order parameter, Physica D 72 (1994) 287-308.

18. E. Fried, Continua described by a microstructural field, Zeitschrift für angewandte Mathematik und Physik 47 (1996) 168-175.

19. E. Fried \& S. Sellers, Microforces and the theory of solute transport, Zeitschrift für angewandte Mathematik und Physik, in press.

20. M. E. Gurtin, An Introduction to Continuum Mechanics. New York, London: Academic Press, 1981.

21. C. Truesdell, Rational Thermodynamics, Second Edition. New York, Berlin, Heidelberg, Tokyo: Springer-Verlag, 1984.

22. B. D. Coleman \& W. Noll, The thermodynamics of elastic materials with heat conduction and viscosity, Archive for Rational Mechanics and Analysis 13 (1963) 167-178.

23. M. E. Gurtin \& P. W. Voorhees, The continuum mechanics of coherent twophase elastic solids with mass transport. Proceedings of the Royal Society of London A 444 (1993) 323-343. 
24. W. Nernst, Zur Kinetik der in Lösung befindlichen Körper: I. Theorie der Diffusion, Zeitschrift für physikalische Chemie 2 (1888) 613-637.

25. A. Einstein, Über die von der molekularkinetischen Theorie der Wärme geforderten Bewegung von in ruhenden Flüssigkeiten suspendierten Teilchen, Annalen der Physik 17 (1905) 549-560. 